Pacific Journal of Mathematics

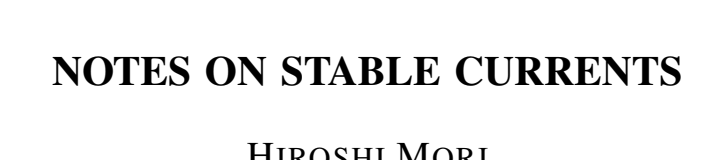




\section{NOTES ON STABLE CURRENTS}

\section{HIROSHI MORI}

\section{With additional assumptions we answer a conjecture pro- posed by Lawson and Simons.}

In a work [5], H. B. Lawson, Jr. and J. Simons proved that there exist no stable rectifiable currents on an $n$-dimensional unit sphere $S^{n}$ in the $(n+1)$-dimensional Euclidean space $R^{n+1}$. And concerning to this fact, they conjectured as follows.

Conjecture. Let $M$ be a compact, simply-connected Riemannian manifold with the sectional curvature satisfying $1 / 4<K_{\delta} \leqq 1$ for all tangent two planes $\sigma$. Then there exist no stable rectifiable currents on $M$.

We obtain the following results with respect to this conjecture.

Let $M$ be a compact, connected $n$-dimensional Riemannian manifold isometrically immersed in $(n+1)$-dimensional Euclidean space $R^{n+1}$. Let $\delta$ be a constant with $0<\delta \leqq 1$, and suppose that at each point $x$ of $M$, with respect to a suitable unit normal, every principal curvature $\lambda_{j}$ of $M$ satisfies

$$
\sqrt{\delta} \leqq \lambda_{j} \leqq 1
$$

$j=1, \cdots, n$.

THEOREM. Let $M$ be a compact, connected Riemannian manifold satisfying the conditions expressed above. Associate to each $\mathscr{S} \rightarrow$ $\mathscr{R}_{p}(M)$ a quadratic form $Q_{S}$ on $\mathscr{V}$ as follows. For $V \in \mathscr{Y}$, let $\phi_{t}$ be the flow generated by $V$ and set

$$
Q_{\mathscr{S}}(V)=\frac{d^{2}}{d t^{2}} \boldsymbol{M}\left(\phi_{t \sharp} \mathscr{S}\right)_{\mid t=0} .
$$

Then for all $\mathscr{S} \in \mathscr{R}_{p}(M)$

$$
\operatorname{tr} Q_{\mathscr{S}} \leqq p(p+1-n \delta-\delta) \boldsymbol{M}(\mathscr{S}) .
$$

(For definitions of $\mathscr{V}$ and $\mathscr{R}_{p}(M)$, see below.)

COROLlaRY 1. Under the assumptions of the Theorem, for all $p$ with $1 \leqq p<n \delta+\delta-1$, any rectifiable $p$-current $\mathscr{S} \leftarrow \mathscr{R}_{p}(M)$ is not stable. If $\delta$ satisfies $n /(n+1)<\delta \leqq 1$, then any rectifiable p-current $\mathscr{S} \in \mathscr{R}_{p}(M)$ is not stable for each $p$ with $1 \leqq p \leqq n-1$. 
CoRollary 2. Under the assumptions of the Theorem, if $\delta$ satisfies $n /(n+1)<\delta \leqq 1$, then

$$
H_{p}(M ; Z)=H_{p}\left(S^{n} ; Z\right)
$$

for each $p$ with $0 \leqq p \leqq n$. Therefore, in particular, if $n=2$ or $n \geqq 5$, then $M$ is homeomorphic to $S^{n}$. (This conclusion follows from weaker conditions.)

The author is grateful to Professor H. Kitahara for his many valuable suggestions in this research. The author also wishes to thank Mr. Maeda for his kind advice.

1. In the following, we use the same notation as in [5]. Also see [5] for detailed definitions. Let $M$ be a compact $n$-dimensional Riemannian manifold with Riemannian metric $g$ and canonical connection $\nabla$. For a point $x \in M, T_{x}(M)$ denotes the tangent space of $M$ at $x$. Let $\mathscr{R}_{p}(M)$ be the set of all rectifiable $p$-currents on $M$, where $0 \leqq p \leqq n$. For a current $\mathscr{S} \in \mathscr{R}_{p}(M), \overrightarrow{\mathscr{S}}_{x}$ denotes an orientation of $\mathscr{S}$, that is, for $\mathscr{H}^{p}$-almost all $x \in \mathscr{S}, \overrightarrow{\mathscr{S}}_{x}$ is a simple $p$-vector of unit length which represents $T_{x}(\mathscr{S})$, where $\mathscr{H}^{p}$ is the Hausdorff $p$-measure on $M$. Let $V$ be a smooth vector field on $M$. We define a linear mapping $\mathscr{A}^{v}: T_{x}(M) \rightarrow T_{x}(M)$ by $\mathscr{A}^{v}(X):=\nabla_{X} V$ for $X \in T_{x}(M)$. This mapping can be extended uniquely as a derivation to $\Lambda^{p} T_{x}(M)$, that is, as a linear map $\mathscr{A}^{v}: \bigwedge^{p} T_{x}(M) \rightarrow \bigwedge^{p} T_{x}(M)$ which for simple vectors is given by

$\mathscr{A}^{v}\left(X_{1} \wedge \cdots \wedge X_{p}\right)=\sum_{i=1}^{p} X_{1} \wedge \cdots \wedge X_{i-1} \wedge \mathscr{A}^{v} X_{i} \wedge X_{i+1} \wedge \cdots \wedge X_{p}$

At $x \in M$, we define also the linear map $\nabla_{V}, \cdot V: T_{x}(M) \rightarrow T_{x}(M)$ by $\Delta_{V, X} V:=\nabla_{V} \nabla_{\tilde{X}} V-\nabla_{\Delta_{V} \check{X}} V$ for $X \in T_{x}(M)$, where $\tilde{X}$ is any extension of $X$ to a local vector field. The definition is independent of any extension $\tilde{X}$, and the map carries over uniquely as a derivation to $\bigwedge^{p} T_{x}(M)$. Consider a current $\mathscr{S} \in \mathscr{R}_{p}(M)$ and a vector field $V$ on $M$. Let $\phi_{t}: M \rightarrow M, t \in R$ be the 1-parameter group of diffeomorphisms generated by $V$. Then for each $t \in R$ we have the rectifiable current $\phi_{t \sharp}(\mathscr{S})$ which, as a linear functional on $\Lambda^{p}(M)$, is given by

$$
\left(\phi_{t \sharp} \mathscr{S}\right)(\omega)=\mathscr{S}\left(\phi_{t}^{*} \omega\right)
$$

for $\omega \in \Lambda^{p}(M)$, where $\Lambda^{p}(M)$ is the space of all smooth $p$-forms on $M$. Let $M$ denote the usual norm of a linear functional on $\Lambda^{p}(M)$ which has the usual Fréchet topology. Then,

$$
\left.\boldsymbol{M}\left(\phi_{t \sharp} \mathscr{S}\right)=\int_{\boldsymbol{M}} \sqrt{\left(\phi_{t}^{*} g\right)(\overrightarrow{\mathscr{S}}, \overrightarrow{\mathscr{S}})} d\|\mathscr{S}\|\right)
$$


where $\|\mathscr{S}\|$ is a measure on $M$ defined, by using the $p$-dimensional Hausdorff measure $\mathscr{C}^{p}$ on $M$, as follows: for a Borel set $X \subset M$, $\|\mathscr{S}\|(X)=\mathscr{H}^{p}(X \cap \mathscr{S})$.

Definition. A rectifiable $p$-current $\mathscr{S} \in \mathscr{R}_{p}(M)$ is said to be stable if, for each vector field $V$ the following two conditions hold:

$$
\begin{aligned}
& \frac{d}{d t} \boldsymbol{M}\left(\dot{\phi}_{t \sharp} \mathscr{S}\right)_{\mid t=0}=0, \\
& \frac{d^{2}}{d t^{2}} \boldsymbol{M}\left(\dot{\phi}_{t \sharp} \mathscr{S}\right)_{\mid t=0} \geqq 0 .
\end{aligned}
$$

The following is obtained by Lawson and Simons in [5].

Proposition 1. Let $M$ be a compact Riemannian manifold and $V$ a vector field on $M$ with associated flow $\phi_{t}$. Then for any rectifiable p-current $\mathscr{S} \in \mathscr{R}_{p}(M)$,

$$
\begin{gathered}
\frac{d}{d t} \boldsymbol{M}\left(\phi_{t \sharp} \mathscr{S}\right)_{\mid t=0}=\int_{M}\langle\mathscr{\mathscr { A }} \overrightarrow{\mathscr{S}}, \overrightarrow{\mathscr{S}}\rangle d\|\mathscr{S}\|, \\
\frac{d^{2}}{d t^{2}} \boldsymbol{M}\left(\dot{\phi}_{t \sharp} \mathscr{S}\right)_{\mid t=0}=\int_{M}\left\{-\left\langle\mathscr{S}^{V} \overrightarrow{\mathscr{S}}, \overrightarrow{\mathscr{S}}\right\rangle^{2}+\left\langle\mathscr{S}^{V} \mathscr{\mathscr { S }}^{V}(\overrightarrow{\mathscr{S}}), \overrightarrow{\mathscr{S}}\right\rangle\right. \\
\left.+\left|\mathscr{A}^{V}(\overrightarrow{\mathscr{S}})\right|^{2}+\left\langle\nabla_{V, \overrightarrow{\mathscr{S}}} V, \overrightarrow{\mathscr{S}}\right\rangle\right\} d\|\mathscr{S}\| .
\end{gathered}
$$

REMARK. In the special case that $V=\nabla f(=$ the gradient of $f$ ) for some $f \in C^{3}(M)$, the transformation $\mathscr{C}^{V}$ is symmetric and (2) simplifies to

$$
\begin{aligned}
& \frac{d^{2}}{d t^{2}} \boldsymbol{M}\left(\phi_{t \sharp \mathscr{S}} \mathscr{S}\right)_{\mid t=0}=\int_{M}\left\{-\left\langle\mathscr{A}^{V} \overrightarrow{\mathscr{S}, \mathscr{S}}\right\rangle^{2}+2\left|\mathscr{\mathscr { S }}^{V}(\mathscr{S})\right|^{2}\right. \\
& \quad+\langle\nabla, \overrightarrow{\mathscr{S}} V, \mathscr{S}\rangle\} d\|\mathscr{S}\| .
\end{aligned}
$$

For future reference we shall write the integrand of $(2)^{\prime}$ at $x \in$ $M$ in terms of tangent vectors at $x$. Let $\left\{\bar{e}_{1}, \cdots, \bar{e}_{p}, \bar{e}_{p+1}, \cdots, \bar{e}_{n}\right\}$ be an orthonormal basis of $T_{x}(M)$ and set $\xi=\bar{e}_{1} \wedge \cdots \wedge \bar{e}_{p}$. Then

$$
\begin{aligned}
&-\left\langle\mathscr{L}^{V} \xi, \xi\right\rangle^{2}+2\left|\mathscr{A}^{V}(\xi)\right|^{2}+\left\langle\nabla_{V, \xi} V, \xi\right\rangle \\
&=\left\{\sum_{j=1}^{p}\left\langle\mathscr{A}^{V}\left(\bar{e}_{j}\right), \bar{e}_{j}\right\rangle\right\}^{2}+2 \sum_{j=1}^{p} \sum_{\alpha=p+1}^{n}\left\langle\mathscr{C}^{V}\left(\bar{e}_{j}\right), \bar{e}_{\alpha}\right\rangle^{2} \\
&+\sum_{i=1}^{p}\left\langle\nabla_{V, \bar{e}_{j}} V, \bar{e}_{j}\right\rangle,
\end{aligned}
$$

where $\left|\mathscr{A}^{V}(\xi)\right|$ denotes the length of $p$-vector $\mathscr{A}^{V}(\xi)$.

2. Now we assume that $M$ is isometrically immersed in $(n+1)$ - 
dimensional Euclidean space $R^{n+1}$ with canonical Riemannian metric $\langle$,$\rangle and canonical Riemannian connection \bar{\nabla}$. For all local formulas we may consider the isometric immersion $f$ of $M$ into $R^{n+1}$ as an imbedding and thus identify $x \in M$ with $f(x) \in R^{n+1}$. The tangent space $T_{x}(M)$ is identified with a subspace of the tangent space $T_{x}\left(R^{n+1}\right)$. The normal space $T_{x}^{\perp}$ is the subspace of $T_{x}\left(R^{n+1}\right)$ consisting of all $\zeta \epsilon$ $T_{x}\left(R^{n+1}\right)$ which are orthogonal to $T_{x}(M)$ with respect to the Riemannian metric $\langle$,$\rangle . For each point x$ of $M$, choose a field $\zeta$ of unit normal vectors defined on a neighborhood $U$ of $x$. Then we have the basic formulas

$$
\begin{aligned}
& \bar{\nabla}_{X} Y=\nabla_{X} Y+\left\langle A_{\zeta} X, Y\right\rangle \zeta \\
& \bar{\nabla}_{X} \zeta=-A_{\zeta} X
\end{aligned}
$$

where $X$ and $Y$ are smooth vector fields tangent to $M$, and $A_{\zeta}$ is a tensor field of type $(1,1)$, called the second fundamental form associated with $\zeta$. The Gauss equation expresses the curvature tensor $R$ of $M$ as follows.

$$
R(X, Y) Z=\left\langle A_{\zeta} Y, Z\right\rangle A_{\zeta} X-\left\langle A_{\zeta} X, Z\right\rangle A_{\zeta} Y
$$

where $X, Y$ and $Z$ are smooth vector fields tangent to $M$.

Let $\delta$ be a constant with $0<\delta \leqq 1$, and suppose that at each point $x$ of $M$, with respect to a suitable field $\zeta$ of unit normals, every principal curvature $\lambda_{j}$ of $M$ satisfies $\sqrt{\delta} \leqq \lambda_{j} \leqq 1, j=1, \cdots, n$.

REMARK. The above assumption implies that $M$ has the sectional curvature satisfying $\delta \leqq K_{\delta} \leqq 1$ for all tangent two planes $\sigma$. And from the continuity of the eigen-values of the linear map $A_{\zeta}: T_{x}(M) \rightarrow$ $T_{x}(M)$, called the principal curvatures of $M$, the above assumption also implies that $M$ is orientable. Therefore we can choose a global field $\zeta$ of unit normals on $M$ which satisfies the above condition, and then we can write $A_{\zeta}=A$.

3. To estimate the left hand side of $\left(\mathrm{s}_{2}\right)$ we begin with the space of functions $\mathscr{F}=\left\{\psi \mid M ; \psi: R^{n+1} \rightarrow R\right.$ is linear $\}$, and define

$$
\mathscr{C}=\{\nabla \psi ; \psi \in \mathscr{F}\} \text {. }
$$

Then there is a natural isomorphism

$$
\mathscr{V} \cong R^{n+1}
$$

which associates to $v \in R^{n+1}$ the gradient of the function $\psi_{v}(x)=\langle v, x\rangle$ on $M$. This identification introduces a natural inner product on $\mathscr{V}$.

To any simple unit $p$-vector $\xi \in \Lambda^{p} T_{x}(M)$, at any $x \in M$, we can associate a quadratic form $Q_{\xi}$ on $\mathscr{V}$ as follows. For $V \in \mathscr{Y}$, let $\phi_{t}$ 
be the flow generated by $V$, and define

$$
Q_{\xi}(V)=\frac{d^{2}}{d t^{2}}\left|\phi_{t \sharp} \xi\right|_{1 t=0} .
$$

Then we have the following.

Proposition 2. Under the assumptions as expresses above, we have

$$
\operatorname{tr} Q_{\xi} \leqq p(p+1-n \delta-\delta)
$$

Proof. Suppose $V \in \mathscr{V}$ corresponds to $v \in R^{n+1}$ under the isomorphism (4). Then at any $y \in M$

$$
V(y)=v-\left\langle v, \zeta_{y}\right\rangle \zeta_{y},
$$

and then for $X \in T_{x}(M), \nabla_{X} V=\left(\bar{V}_{X} V\right)^{T}=\left\langle v, \zeta_{x}\right\rangle A X$, where ()$^{T}$ denotes orthogonal projection $T_{x}\left(R^{n+1}\right) \rightarrow T_{x}(M)$. Thus,

$$
\mathscr{A}^{v}(X)=\nabla_{X} V=\left\langle v, \zeta_{x}\right\rangle A X \text {. }
$$

And it follows easily that

$$
\nabla_{V, X} V=-\langle V, A V\rangle A X+\left\langle v, \zeta_{x}\right\rangle \nabla_{V}(A \tilde{X})-\left\langle v, \zeta_{x}\right\rangle A\left(\nabla_{V} \tilde{X}\right)
$$

where $\tilde{X}$ is any extension of $X$ to a local vector field.

We now choose an orthonormal basis $\left\{x_{0}=\zeta_{x}, x_{1}=e_{1}, \cdots, x_{n}=e_{n}\right\}$ for $R^{n+1}$, where $e_{j}$ is an eigenvector corresponding to the eigenvalue $\lambda_{j}$ of $A, j=1, \cdots, n$. Via (4) this fixes an orthonormal basis $\left\{V_{0}\right.$, $\left.V_{1}, \cdots, V_{n}\right\}$ of $\mathscr{V}$. It then follows from (5) and (6) that $\nabla_{V_{0}}, \cdot V_{0}=$ $\mathscr{A}^{v} 1=\cdots=\mathscr{A}^{v} n=0$ and $\mathscr{A}^{v} o=A, \nabla_{V_{j}}, \cdot V_{j}=-\lambda_{j} A, j=1, \cdots n$, as transformations of $T_{x}(M)$. For given simple unit $p$-vector $\xi \in$ $\bigwedge^{p} T_{x}(M)$, we can choose an orthonormal basis $\left\{\bar{e}_{1}, \cdots, \bar{e}_{p}, \bar{e}_{p+1}, \cdots, \bar{e}_{n}\right\}$ of $T_{x}(M)$ with $\xi=\bar{e}_{1} \wedge \cdots \wedge \bar{e}_{p}$. It then follows from (2), (2)', (3) and above formulas that

$$
\begin{aligned}
\operatorname{tr}\left(Q_{\xi}\right)= & \sum_{l=0}^{n} Q_{\xi}\left(V_{l}\right) \\
= & \sum_{l=0}^{n}\left\{\left(\sum_{j=1}^{p}\left\langle\mathscr{A}^{v} l \bar{e}_{j}, \bar{e}_{j}\right\rangle\right)^{2}+2 \sum_{j=1}^{p} \sum_{\alpha=p+1}^{n}\left\langle\mathscr{A}^{v} l \bar{e}_{j}, \bar{e}_{\alpha}\right\rangle^{2}\right. \\
& \left.+\sum_{j=1}^{p}\left\langle\nabla_{V_{l}, \bar{e}_{j}} V_{l}, \bar{e}_{j}\right\rangle\right\} \\
= & \left(\sum_{j=1}^{p}\left\langle A \bar{e}_{j}, \bar{e}_{j}\right\rangle\right)^{2}+2 \sum_{j=1}^{p} \sum_{\alpha=p+1}^{n}\left\langle A \bar{e}_{j}, \bar{e}_{\alpha}\right\rangle^{2}-\sum_{l=1}^{n} \sum_{j=1}^{p}\left\langle\lambda_{l} A \bar{e}_{j}, \bar{e}_{j}\right\rangle \\
= & \left(\sum_{j=1}^{p}\left\langle A \bar{e}_{j}, \bar{e}_{j}\right\rangle\right)^{2}+2 \sum_{j=1}^{p}\left(\left|A \bar{e}_{j}\right|^{2}-\sum_{i=1}^{p}\left\langle A \bar{e}_{j}, \bar{e}_{i}\right\rangle^{2}\right) \\
& -\sum_{l=1}^{n} \sum_{j=1}^{p} \lambda_{l}\left\langle A \bar{e}_{j}, \bar{e}_{j}\right\rangle
\end{aligned}
$$




$$
\begin{aligned}
= & 2 \sum_{j=1}^{p}\left|A \bar{e}_{j}\right|^{2}+\sum_{\substack{i, j=1 \\
i \neq j}}^{p}\left(\left\langle A \bar{e}_{i}, \bar{e}_{i}\right\rangle\left\langle A \bar{e}_{j}, \bar{e}_{j}\right\rangle-2\left\langle A \bar{e}_{j}, \bar{e}_{i}\right\rangle^{2}\right) \\
& -\sum_{j=1}^{p}\left\langle A \bar{e}_{j}, \bar{e}_{j}\right\rangle^{2}-\sum_{l=1}^{n} \sum_{j=1}^{p} \lambda_{l}\left\langle A \bar{e}_{j}, \bar{e}_{j}\right\rangle .
\end{aligned}
$$

By the assumption, $\sqrt{\delta} \leqq \lambda_{j} \leqq 1, j=1, j=1, \cdots, n$, we get $\left|A \bar{e}_{j}\right|^{2} \leqq 1$, and $V \bar{\delta} \leqq\left\langle A \bar{e}_{j}, \bar{e}_{j}\right\rangle \leqq 1$ for $1, \cdots, n$. Thus we have

$$
\begin{aligned}
\operatorname{tr}\left(Q_{\xi}\right) & \leqq 2 p+p(p-1)-p \delta-n p \delta \\
& =p(p+1-n \delta-\delta)
\end{aligned}
$$

Combining Proposition 1 and Proposition 2 we get the theorem and the Corollary 1. And by virtue of the basic theorems on integral currents, we have the Corollary 2, see [2] or [5].

\section{REFERENCES}

1. H. Federer, Geometric Measure Theory, Springer-Verlag, New York, 1969.

2. H. Federer and W. Fleming Normal and integral currents, Ann. of Math., 72 (1960), 458-520.

3. S. Kobayashi and K. Nomizu, Foundations of Differential Geometry, Vol. II, Interscience, New York, 1969.

4. H. B. Lawson, Jr., Minimal Varieties in Real and Complex Geometry, University of Montréal Press, Montréal, 1973.

5. H. B. Lawson, Jr. and J. Simons, On the stable currents and their application to global problems in real and complex geometry, Ann. of Math., 98 (1973), 427-450.

6. M. Maeda, The formula of second variation and the injectivity radius of certain manifolds, to appear.

7. J. Milnor, Lectures on Morse theory, Ann. Math. Studies No. 51, Princeton University Press, Princeton, New Jersey, 1963.

Received March 28, 1975.

TOYAMa UnIVERSITY 


\section{PACIFIC JOURNAL OF MATHEMATICS}

\section{EDITORS}

RICHARD ARENS (Managing Editor) University of California

Los Angeles, California 90024

\section{R. A. BEAUMONT}

University of Washington Seattle, Washington 98105
J. DugundjI Department of Mathematics University of Southern Californı Los Angeles, California 90007

D. Gilbarg AND J. Milgram Stanford University Stanford, California 94305

\section{ASSOCIATE EDITORS}

E. F. BECKENBACH

B. H. NeUMANN

F. WOLF

K. YosHidA

\section{SUPPORTING INSTITUTIONS}

UNIVERSITY OF BRITISH COLUMBIA CALIFORNIA INSTITUTE OF TECHNOLOGY UNIVERSITY OF CALIFORNIA MONTANA STATE UNIVERSITY UNIVERSITY OF NEVADA NEW MEXICO STATE UNIVERSITY OREGON STATE UNIVERSITY UNIVERSITY OF OREGON OSAKA UNIVERSITY

\author{
UNIVERSITY OF SOUTHERN CALIFORNIA \\ STANFORD UNIVERSITY \\ UNIVERSITY OF TOKYO \\ UNIVERSITY OF UTAH \\ WASHINGTON STATE UNIVERSITY \\ UNIVERSITY OF WASHINGTON \\ AMERICAN MATHEMATICAL SOCIETY \\ NAVAL WEAPONS CENTER
}




\section{Pacific Journal of Mathematics}

\section{Vol. 61, No. $1 \quad$ November, 1975}

Jiří Adámek, V. Koubek and Věra Trnková, Sums of Boolean spaces represent every

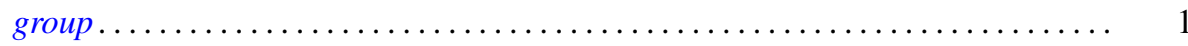

Richard Neal Ball, Full convex l-subgroups and the existence of $a^{*}$-closures of

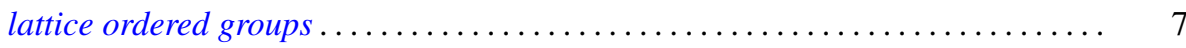

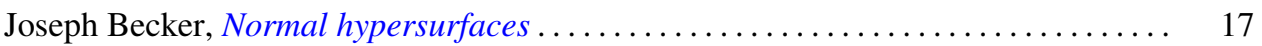

Gerald A. Beer, Starshaped sets and the Hausdorff metric . . . . . . . . . . . . . 21

Dennis Dale Berkey and Alan Cecil Lazer, Linear differential systems with

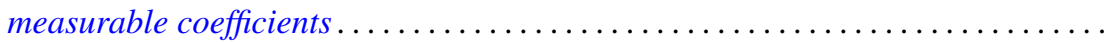

Harald Boehme, Glättungen von Abbildungen 3-dimensionaler

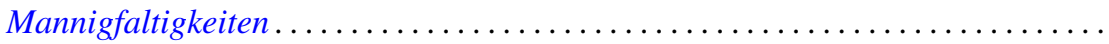

Stephen LaVern Campbell, Linear operators for which $T^{*} T$ and $T+T^{*}$

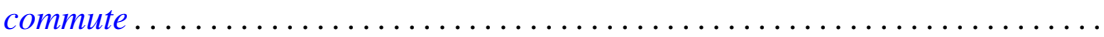

H. P. Dikshit and Arun Kumar, Absolute summability of Fourier series with

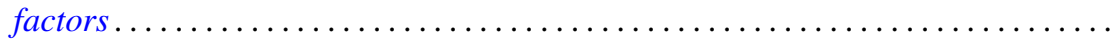

Andrew George Earnest and John Sollion Hsia, Spinor norms of local integral

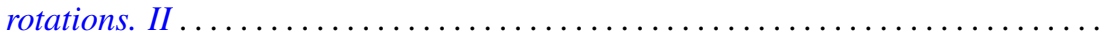

Erik Maurice Ellentuck, Semigroups, Horn sentences and isolic structures .........

Ingrid Fotino, Generalized convolution ring of arithmetic functions . . . . . . . . . . .

Michael Randy Gabel, Lower bounds on the stable range of polynomial rings .......

Fergus John Gaines, Kato-Taussky-Wielandt commutator relations and

characteristic curves

Theodore William Gamelin, The polynomial hulls of certain subsets of $C^{2}$

R. J. Gazik and Darrell Conley Kent, Coarse uniform convergence spaces. . .

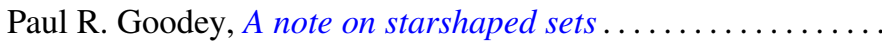

Eloise A. Hamann, On power-invariance

M. Jayachandran and M. Rajagopalan, Scattered compactification for $N \cup\{P\}$. . .

V. Karunakaran, Certain classes of regular univalent functions .

John Cronan Kieffer, A ratio limit theorem for a strongly subadditive set function in a locally compact amenable group .................

Siu Kwong Lo and Harald G. Niederreiter, Banach-Buck measure, density, and uniform distribution in rings of algebraic integers ........

Harold W. Martin, Contractibility of topological spaces onto metric spaces ....

Harold W. Martin, Local connectedness in developable spaces .

A. Meir and John W. Moon, Relations between packing and covering numbers of a tree.

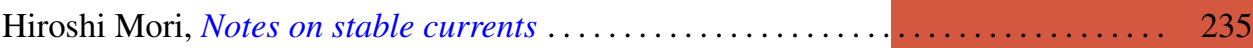

Donald J. Newman and I. J. Schoenberg, Splines and the logarithmic function . . . . 241

M. Ann Piech, Locality of the number of particles operator....

Fred Richman, The constructive theory of $K T$-modules .......

Gerard Sierksma, Carathéodory and Helly-numbers of

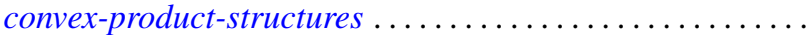

Raymond Earl Smithson, Subcontinuity for multifunctions .... . . 